\title{
GENOTYPE IDENTIFICATION OF HEPATITIS C VIRUS (HCV) ISOLATED FROM A SINGLE JAPANESE CARRIER IN NAGASAKI PREFECTURE AND GENOME ANALYSIS OF E1 AND E2/NS1 ENVELOPE GLYCOPROTEIN REGIONS
}

\author{
WEI-YUN ZHENG \\ Received September 20, 1994/Accepted October 30, 1994
}

\begin{abstract}
The nucleotide (nt) sequence of a Hepatitis $\mathrm{C}$ virus genome ( $\mathrm{HCV}-\mathrm{N}$ ) which was derived from a single Japanese patient's serum in Nagasaki Prefecture has been determined by multiple clones coverning 22 overlapping regions of the HCV geneme. The sequenced region consisted of $9295 \mathrm{nt}$, including $248 \mathrm{nt}$ of 5'-untranslated region (UTR), a single large open reading frame (ORF) encoding a polyprotein of 3010 amino acids (aa) and a 17 nt of 3'-UTR. Phylogenetic analysis indicated that HCV-N belongs to II/1b genotype of group 1. Two other Nagasaki HCV strains (HCV-N1 and HCV-N2) were also sequenced in the $\mathrm{E} 1$ and $\mathrm{N}$-terminus of the E2/NS1 regions. Two hypervariable regions (HVR 1 and HVR 2) were found in the N-terminus of E2/NS1 region among 3 Nagasaki strains and 7 other HCV strains with published sequences. Two well-conserved aa sequences were also identified among $10 \mathrm{HCV}$ strains in the E1 and $\mathrm{N}$ -terminus of the E2/NS1 regions. The results will be useful for future underștanding on the pathogenesis, virological diagnosis and development of vaccine for $\mathrm{HCV}$.
\end{abstract}

\section{INTRODUCTION}

Hepatitis $\mathrm{C}$ virus (HCV) is the major cause of nonA -nonB (NANB) hepatitis, and chronic infection with $\mathrm{HCV}$ has been linked to the development of liver cirrhosis and hepatocellular carcinoma (Plagemann, 1991). Many entire and partial $\mathrm{HCV}$ sequence informations published so far have shown that HCV genome consisted of about $10 \mathrm{~Kb}$ single-stranded positive-sense RNA with 5'UTR, a single long ORF followed by 3'UTR. The viral genome organization resembles that of the flaviviruses and pestiviruses (Kato et al., 1990; Plagemann, 1991).

Since Choo et al. (1989) cloned the genome of an RNA virus from the plasma of a chimpanzee inoculated with plasma from patient with NANB hepatitis and designated it as $\mathrm{HCV}$, entire sequences have been reported for at least $15 \mathrm{HCV}$ strains. There are: $\mathrm{HCV}-\mathrm{J}$ (Kato et al., 1990), HCV-1 (Choo et al., 1991), HCV-H (Inchauspe et al., 1991), HCV-BK (Takamizawa et al., 1991), HC-J6 (Okamoto et al., 1991), HC-J1 (Okamoto et al., 1992a), HC-J8 (Okamoto et al., 1992b), HC-J4/ 83, HC-J4/91 (Okamoto et al., 1992c), HCV-JT (Tana- ka et al., 1992), HCV-T (Chen et al., 1992), HCV-JK1 (Honda et al., 1993), HC-C2 (Wang et al., 1993), HC-G9 (Okamoto et al., 1994) and NZL1 (Sakamoto et al., 1994). Depending on the HCV sequence similarity, $\mathrm{HCV}$ genome can be classified into 6 genotypes: I, II, III, IV, $\mathrm{V}$ and VI as reviewed by Sakamoto et al. (1994), or into 6 major genotypes: 1 (a, b, c), 2 (a, b, c), 3 (a, b) , 4, 5 and 6 (Simmonds et al., 1933b).

Recently, the newest classification has been summarized by Sakamoto et al. (1994) who classified HCV genome into 3 major groups with entire published sequences. The entire HCV sequence of Japanese strains have been assigned either to group 1 (I/1a genotype: $\mathrm{HC}-\mathrm{J} 1$; II/1b genotype: $\mathrm{HCV}-\mathrm{J}, \mathrm{HCV}-\mathrm{BK}, \mathrm{HC}$ -J4/83, HC-J4/91, HCV-JT and HCV-JK1) or group 2 (III/2a genotype: HC-J6; IV/2b genotype: HC-J8) according to Sakamoto et al. (1994). In order to know whether there is particular HCV in the local area of Nagasaki, Japan, HCV patient serum which came from Nagasaki Prefecture was obtained and the genome sequence of the $\mathrm{HCV}-\mathrm{N}$ in this serum was determined by multiple overlapping clones. 
In order to see the genetic variability of $\mathrm{HCV}$ in the Nagasaki area, $\mathrm{HCV}$ genome sequence in 2 more $\mathrm{HCV}$ patient sera in Nagasaki Prefecture (HCV-N1 and $\mathrm{HCV}-\mathrm{N} 2$ ) were also analyzed similarly in the $\mathrm{E} 1$ and $\mathrm{N}$ -terminus of the E2/NS1 envelope glycoprotein regions. Two hypervariable regions have be found among these 3 Nagasaki HCV strains using the sequence diversity comparison with published sequence data of $7 \mathrm{HCV}$ strains.

\section{MATERIALS AND METHODS}

\section{RNA extraction from HCV patient serum}

Serum samples were obtained from NANB patients in Nagasaki Prefecture which were kindly provided by the Second Department of Internal Medicine of Nagasaki University Hospital. These patients were confirmed to be infected with $\mathrm{HCV}$ by anti-C100 HCV ELISA Kit (Ortho Diagnostic Systems, Tokyo, Japan) and HCV reverse-transcription polymerase chain reaction (RT -PCR) as published by Kurihara (1992). One hundred microliters of HCV patient serum was mixed with $20 \mu \mathrm{l}$ of $10 \%$ sodium dodecyl sulfate (SDS) and $80 \mu \mathrm{l}$ of sterile distilled $\mathrm{H}_{2} \mathrm{O}$ at room temperature for $5 \mathrm{~min}$. HCV RNA was extracted with phenol / chloroform and precipitated with 3 volumes of ice-cold absolute ethanol. After storage at $-80^{\circ} \mathrm{C}$ for $1 \mathrm{hr}, \mathrm{HCV}$ RNA was pelleted in an Eppendolf centrifuge with $15,000 \mathrm{xg}$ at $4^{\circ} \mathrm{C}$ for 30 min. The pellet was washed once in $70 \%$ ethanol, vacuum dried and dissolved in $100 \mu \mathrm{l}$ sterile distilled water. RNA solution was stored at $-80^{\circ} \mathrm{C}$.

Selection and synthesis of oligonucleotide primers

Oligonucleotide primer sequences were selected based on the published sequence data (Choo et al., 1991, Takamizawa et al., 1991) and gene walking method. Oligonucleotide primers were synthesized by Applied Biosystems Model 392 DNA / RNA Synthesizer and confirmed for their purity by ion-exchange gel chromatography (Gen-pack; Waters).

\section{RT-PCR}

Ten microliters of RNA solution were added with $90 \mu 1$ of RT-PCR mixture [100 pmol of each primers, 0.2 $\mathrm{mM}$ deoxynucleoside triphosphate, $10 \mathrm{mM}$ Tris ( $\mathrm{PH} 8$. 9), $1.5 \mathrm{mM} \mathrm{MgCl}_{2}, 80 \mathrm{mM} \mathrm{KCl}, 0.5 \mathrm{mg}$ of bovine serum albumin per $\mathrm{ml}, 0.1 \%$ sodium cholate, $0.1 \%$ Triton $\mathrm{X}$ $-100,10 \mathrm{U}$ of reverse transcriptase (Life Science Inc.) and $2 \mathrm{U}$ of Tth DNA polymerase, a thermostable DNA polymerase (Toyobo Co.)]. The reaction mixture was covered by 2 drops of mineral oil and incubated for 10 min at $53^{\circ} \mathrm{C}$ for RT. PCR amplification $\left(94^{\circ} \mathrm{C}\right.$ for $60 \mathrm{sec}$, $53^{\circ} \mathrm{C}$ for $90 \mathrm{sec}$ and $72^{\circ} \mathrm{C}$ for $120 \mathrm{sec}$ by thermal cycler;
Iwaki Co.) was started immediately after RT and repeated 35 times. cDNA product was subjected to agarose gel electrophoresis and visualized by ethidium bromide staining.

\section{Cloning and sequencing of HCV cDNA product}

The amplified HCV cDNA was excised from agarose gel, phosphorylated with $\mathrm{T} 4$ polynucleotide kinase (Nippon Gene Co.) and blunted with T4 DNA polymerase (Takara Co.). The modified cDNA fragment was ligated into Smal site of pUC19 and transformed into Escherichia coli JM 109 strain. The recombinant pUC19 carrying inserted cDNA fragment was purified with Wizard $^{\mathrm{TM}}$ Minipreps DNA Purification System (U.S. A.). The cDNA fragment sequence was determined in both directions with sense and antisense primers by dideoxy chain termination method using both ${ }^{35} \mathrm{~S}$ radioisotope-labeling (DNA Sequencing Kit Version 2.0, U.S. A.) and fluorescent dye-labeled DNA sequencing system (373A DNA Sequencer, Applied Biosystems). To avoid sequence variability, 3 colones from each RT-PCR product were isolated independently for nucleotide sequence determination.

Analysis and homology comparison in nucleotide and deduced amino acid sequences were carried out for $\mathrm{HCV}-\mathrm{N}$ and 7 other HCV strains with published entire sequence, using a computer system with DNASIS Mac Version 2.2, NEW CD2 system (Hitachi Software Engineering Co., Ltd, 1992).

\section{RESULTS}

\section{The genome characterization of $\mathrm{HCV}-\mathrm{N}$}

Multiple cDNA clones were isolated from altogether 22 overlapping regions which covered almost entire genome of $\mathrm{HCV}-\mathrm{N}$, except extreme 5' and 3' terminals (Fig. 1). For each of the overlapping region, 3 independent cDNA clones were isolated from the RT -PCR product and sequenced. The cleavage site of the polyprotein coded by the ORF of the HCV-N genome was assigned according to the publications by Okamoto et al. (1992b) and Honda et al. (1993). Total length of the sequenced region of $\mathrm{HCV}-\mathrm{N}$ consisted of $9295 \mathrm{nt}$ and 3010 aa, respectively (Fig. 2). The sequenced region can be divided into $248 \mathrm{nt}$ in the 5'UTR, $9030 \mathrm{nt}$ in a single ORF and $17 \mathrm{nt}$ in the 3'UTR, respectvely. The ORF was considered to be translated into C (191 aa), E1 (192 aa), E2/NS1 (346 aa), NS2 (277 aa), NS3 (609 aa), NS4 (398 aa) and NS5 (997 aa) proteins.

Genotype classification and genome homology comparison among $\mathrm{HCV}-\mathrm{N}$ and 7 other $\mathrm{HCV}$ strains with published sequences 


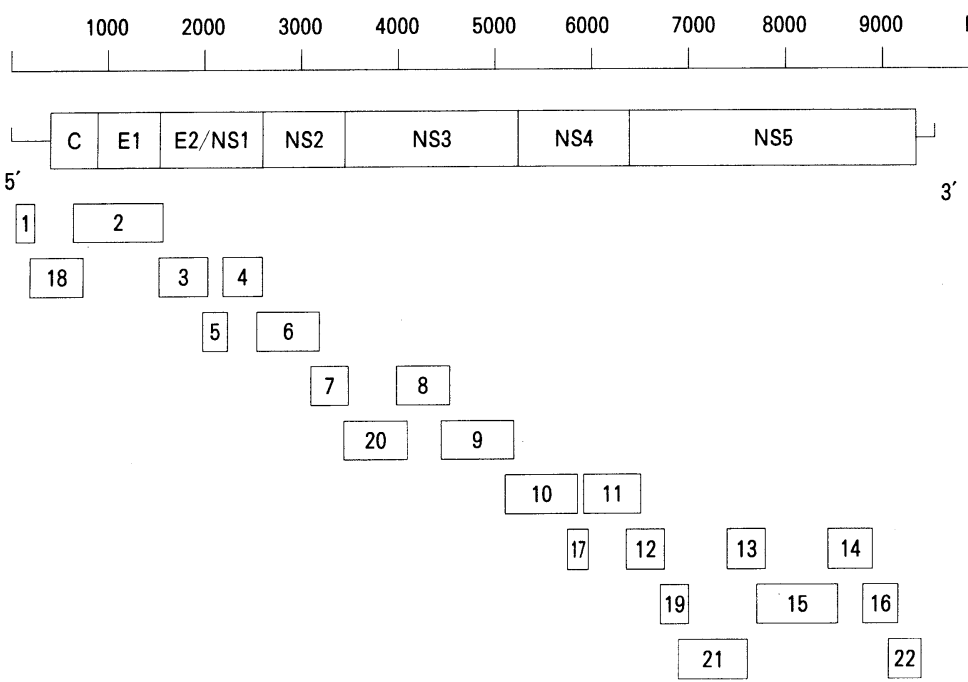

Figure 1 Physical map of the HCV genome and locations of the cDNA clones derived from $\mathrm{HCV}-\mathrm{N}$ strain. Nucleotides are numbered from putative 5'end as indicated on the top. The genome organization of $\mathrm{HCV}$ is according to Okamoto et al. (1994), showing coding region from structural proteins: core (C), envevop (E1 and E2) to nonstructural proteins (NS1-NS5) by blocks, untranslated region (UTR) (5' and 3') by bars. The order of clone number is based on the cloning process.

Entire sequence informations have been published for the following $7 \mathrm{HCV}$ strains: $\mathrm{HCV}-1$ (Choo et al., 1991), HCV-BK (Takamizawa et al., 1991), HCV-J
(Kato et al., 1990), HC-J6 (Okamoto et al., 1991), HC-J8 (Okamoto et al., 1992b), HCV -JT (Tanaka et al., 1992) and HCV-T (Chen et al., 1992). Homology comparison between $\mathrm{HCV}-\mathrm{N}$ and these $7 \mathrm{HCV}$ strains was shown in Table 1. The most conserved region is the 5 UTR which showed nt homology more than 91.5\% among $8 \mathrm{HCV}$ isolates. The sequence homology of the $\mathrm{C}$ protein region was also highly conserved (nt $>81.1 \%$, aa $>88.5 \%$ ). In the putative $\mathrm{E} 1$ and $\mathrm{E} 2 / \mathrm{NS} 1$ regions, the $\mathrm{HCV}-\mathrm{N}$ showed high homology with $\mathrm{HCV}$ -BK, HCV-J, HCV-JT and HCV-T (nt > 84 . $5 \%$, aa $>85.8 \%$ ). Whereas $\mathrm{HCV}-\mathrm{N}$ showed low homology with HC-J6 and HC-J8 (nt < $68.8 \%$, aa $<72.3 \%$ ). Nt sequence homology between $\mathrm{HCV}-\mathrm{N}$ and $\mathrm{HCV}-1$ in the $\mathrm{E} 1$ and E2/NS1 regions was $73.7 \%, 74.5 \%$ and aa sequence homology was $76.0 \%, 81.2 \%$, respectively. From NS2 to NS5 regions, it was evident that $\mathrm{HCV}-\mathrm{N}$ is closer to $\mathrm{HCV}-\mathrm{BK}$, $\mathrm{HCV}-\mathrm{J}, \mathrm{HCV}-\mathrm{JT}$ and $\mathrm{HCV}-\mathrm{T}$ and remote from $\mathrm{HC}-\mathrm{J} 6$ and $\mathrm{HC}-\mathrm{J} 8$, while $\mathrm{HCV}-1$ seems to be located intermediate. The total sequence homology comparison among 8 $\mathrm{HCV}$ isolates gave us a conclusion that $\mathrm{HCV}-\mathrm{N}$ belongs to $\mathrm{HCV}-\mathrm{BK} / \mathrm{HCV}-\mathrm{J} / \mathrm{HCV}-\mathrm{JT} / \mathrm{HCV}-\mathrm{T}$ genotype. In contrast, $\mathrm{HCV}-\mathrm{N}$ is remote from $\mathrm{J} 6$ and $\mathrm{J} 8$ genotypes,

Table 1 Homology comparison of nucleotide and deduced amino acid sequence among 8 HCV strains (HCV-N, HCV-1, HCV-BK, HCV-J, HCV-JT, HCV-T, HC-J6 and $\mathrm{HC}-\mathrm{J} 8$ ). The homology is indicated by $\%$. The nucleotide sequence homology is shown in upper and amino acid sequence homology is shown in lower with parenthesis, respectively.

\begin{tabular}{|c|c|c|c|c|c|c|c|}
\hline Region & $\begin{array}{c}\mathrm{HCV}-\mathrm{N} \\
\& \\
\mathrm{HCV}-1\end{array}$ & $\begin{array}{c}\mathrm{HCV}-\mathrm{N} \\
\& \\
\mathrm{HCV}-\mathrm{BK}\end{array}$ & $\begin{array}{c}\mathrm{HCV}-\mathrm{N} \\
\& \\
\mathrm{HCV}-\mathrm{J}\end{array}$ & $\begin{array}{c}\mathrm{HCV}-\mathrm{N} \\
\& \\
\mathrm{HC}-\mathrm{J} 6\end{array}$ & $\begin{array}{c}\mathrm{HCV}-\mathrm{N} \\
\& \\
\mathrm{HC}-\mathrm{J} 8\end{array}$ & $\begin{array}{c}\mathrm{HCV}-\mathrm{N} \\
\& \\
\mathrm{HCV}-\mathrm{JT}\end{array}$ & $\begin{array}{c}\mathrm{HCV}-\mathrm{N} \\
\& \\
\mathrm{HCV}-\mathrm{T}\end{array}$ \\
\hline Total & $\begin{array}{c}77.9 \\
(84.5)\end{array}$ & $\begin{array}{c}92.0 \\
(94.1)\end{array}$ & $\begin{array}{c}91.5 \\
(93.7)\end{array}$ & $\begin{array}{c}62.4 \\
(70.7) \\
\end{array}$ & $\begin{array}{c}61.7 \\
(69.2) \\
\end{array}$ & $\begin{array}{c}91.7 \\
(93.9) \\
\end{array}$ & $\begin{array}{c}90.0 \\
(92.1) \\
\end{array}$ \\
\hline 5'UTR & 98.8 & 99.2 & 98.4 & 94.0 & 91.5 & 99.2 & 99.2 \\
\hline C & $\begin{array}{c}90.7 \\
(96.3)\end{array}$ & $\begin{array}{c}96.0 \\
(97.4)\end{array}$ & $\begin{array}{c}95.8 \\
(97.9)\end{array}$ & $\begin{array}{c}83.0 \\
(90.1)\end{array}$ & $\begin{array}{c}81.1 \\
(88.5)\end{array}$ & $\begin{array}{c}95.1 \\
(96.3)\end{array}$ & $\begin{array}{c}93.9 \\
(93.7)\end{array}$ \\
\hline E1 & $\begin{array}{c}73.7 \\
(76.0)\end{array}$ & $\begin{array}{c}89.0 \\
(89.6)\end{array}$ & $\begin{array}{c}91.7 \\
(93.2) \\
\end{array}$ & $\begin{array}{c}60.1 \\
(52.9)\end{array}$ & $\begin{array}{c}56.7 \\
(51.8)\end{array}$ & $\begin{array}{c}92.2 \\
(92.2) \\
\end{array}$ & $\begin{array}{c}91.1 \\
(93.8)\end{array}$ \\
\hline $\mathrm{E} 2 / \mathrm{NS} 1$ & $\begin{array}{c}74.5 \\
(81.2)\end{array}$ & $\begin{array}{c}85.7 \\
(88.1)\end{array}$ & $\begin{array}{c}84.5 \\
(86.1)\end{array}$ & $\begin{array}{c}68.8 \\
(71.1)\end{array}$ & $\begin{array}{c}66.8 \\
(72.3)\end{array}$ & $\begin{array}{c}84.7 \\
(85.8)\end{array}$ & $\begin{array}{c}85.3 \\
(87.0) \\
\end{array}$ \\
\hline NS2 & $\begin{array}{c}71.0 \\
(74.4)\end{array}$ & $\begin{array}{c}92.1 \\
(91.7)\end{array}$ & $\begin{array}{c}92.7 \\
(94.9)\end{array}$ & $\begin{array}{c}59.8 \\
(58.7)\end{array}$ & $\begin{array}{c}58.2 \\
(56.2)\end{array}$ & $\begin{array}{c}89.0 \\
(92.4) \\
\end{array}$ & $\begin{array}{c}87.8 \\
(91.3)\end{array}$ \\
\hline NS3 & $\begin{array}{c}78.1 \\
(89.0)\end{array}$ & $\begin{array}{c}90.7 \\
(93.4)\end{array}$ & $\begin{array}{c}90.4 \\
(91.8)\end{array}$ & $\begin{array}{c}69.9 \\
(79.3) \\
\end{array}$ & $\begin{array}{c}69.8 \\
(78.8) \\
\end{array}$ & $\begin{array}{c}91.1 \\
(94.9) \\
\end{array}$ & $\begin{array}{c}89.4 \\
(92.8) \\
\end{array}$ \\
\hline NS4 & $\begin{array}{c}78.9 \\
(88.2)\end{array}$ & $\begin{array}{c}93.1 \\
(97.5)\end{array}$ & $\begin{array}{c}92.7 \\
(97.2)\end{array}$ & $\begin{array}{c}66.8 \\
(74.1)\end{array}$ & $\begin{array}{c}67.0 \\
(72.9)\end{array}$ & $\begin{array}{c}93.5 \\
(97.0) \\
\end{array}$ & $\begin{array}{c}89.9 \\
(94.0)\end{array}$ \\
\hline NS5 & $\begin{array}{c}78.8 \\
(83.7)\end{array}$ & $\begin{array}{c}93.5 \\
(96.3)\end{array}$ & $\begin{array}{c}92.7 \\
(95.2)\end{array}$ & $\begin{array}{c}59.5 \\
(71.8)\end{array}$ & $\begin{array}{c}59.2 \\
(71.5)\end{array}$ & $\begin{array}{c}93.1 \\
(95.2)\end{array}$ & $\begin{array}{c}91.8 \\
(92.5) \\
\end{array}$ \\
\hline
\end{tabular}




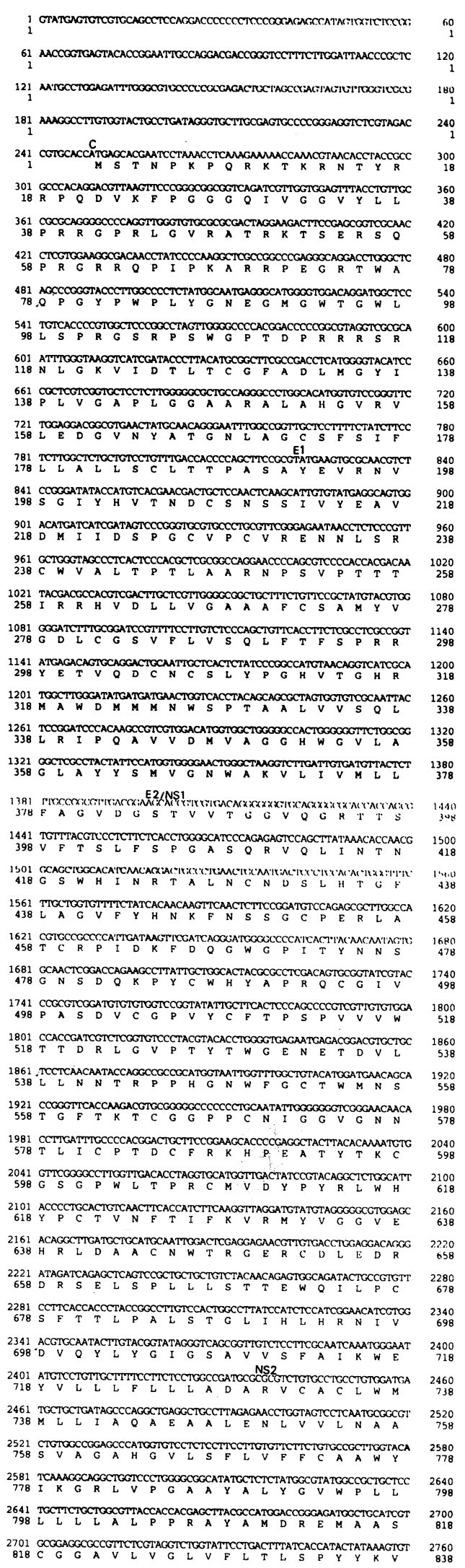

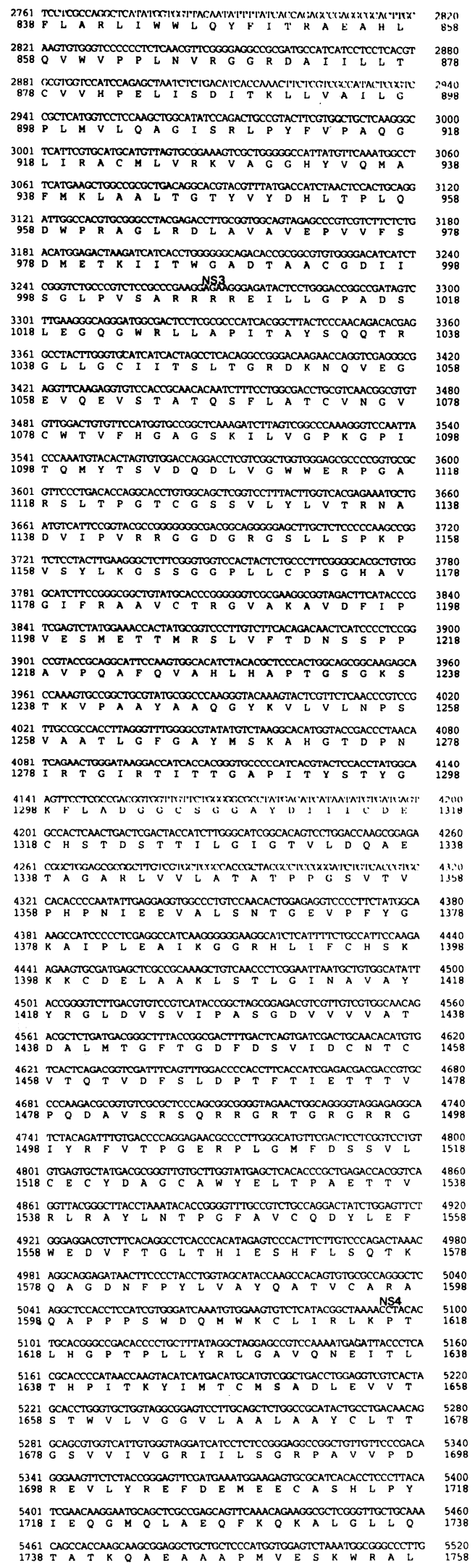

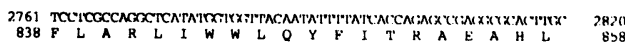

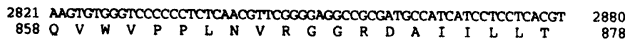

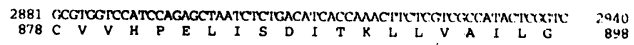

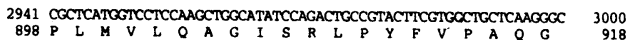

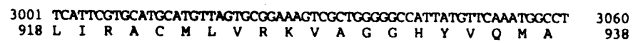

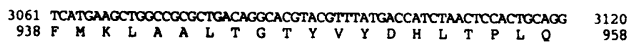

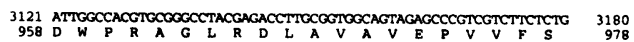

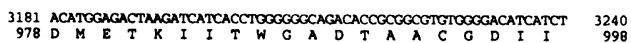

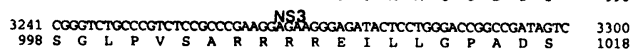

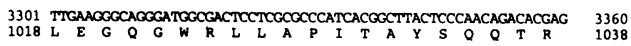

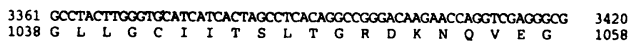

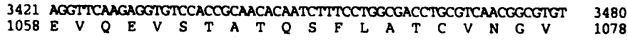

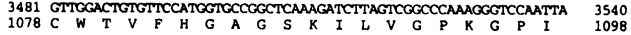

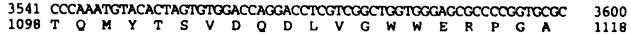

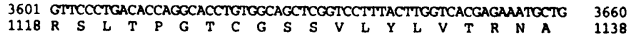

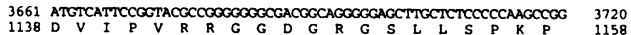

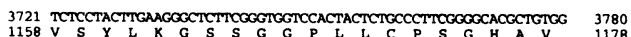

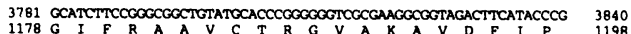

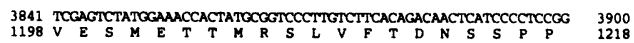

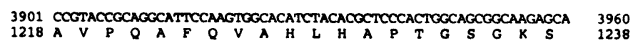

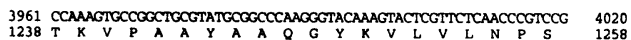

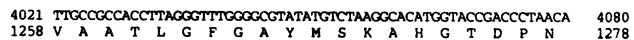

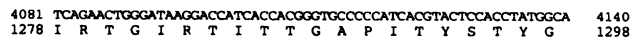

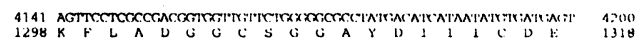

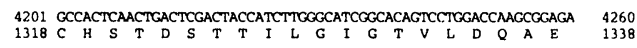

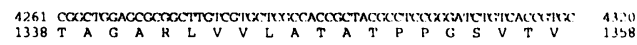

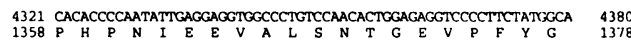

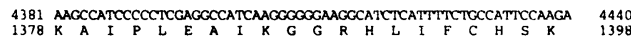

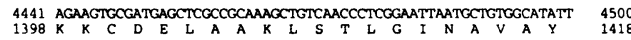

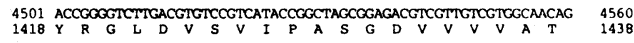

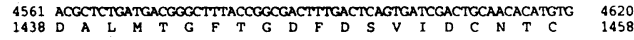
4621
1458

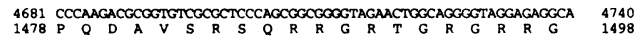

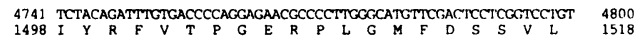

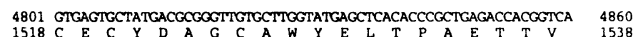

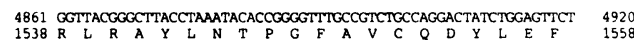

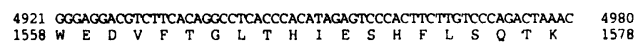

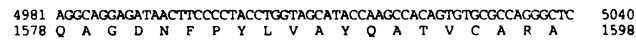

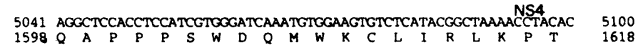

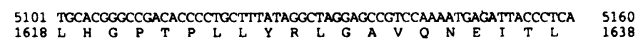

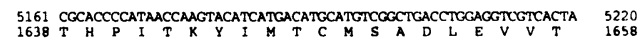

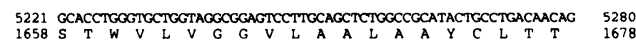

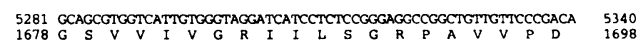

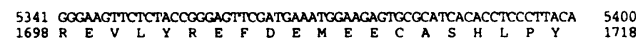

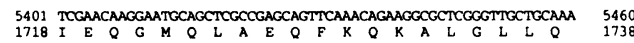

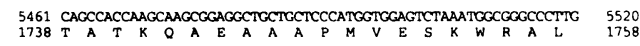

Figure 2 Nucleotide and deduced amino acid sequence of $\mathrm{HCV}-\mathrm{N}$ strain. The nucleotide sequence of $\mathrm{HCV}-\mathrm{N}$ is shown in the upper line and deduced amino acid sequence of $\mathrm{HCV}-\mathrm{N}$ is shown in the lower line, respectively. 


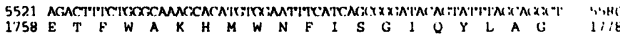

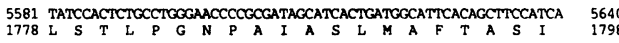

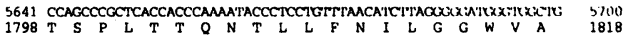

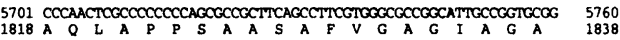

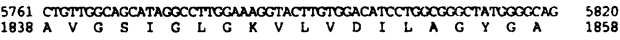

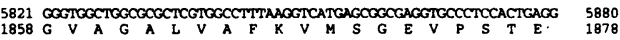

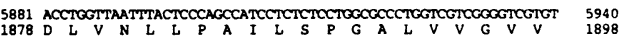

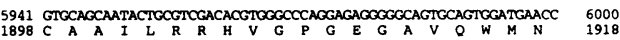

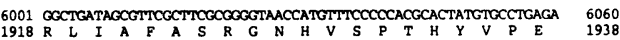

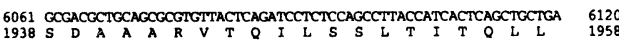

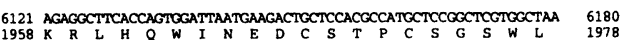

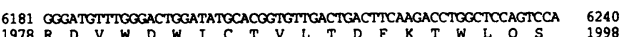

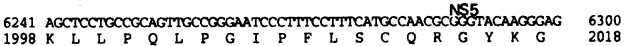

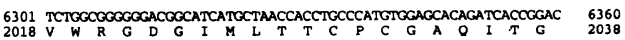

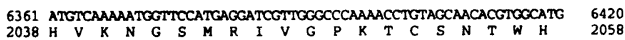

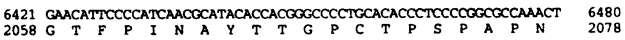

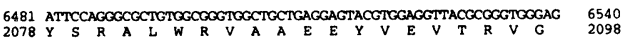

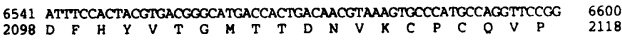

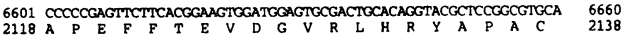

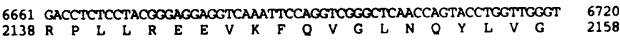

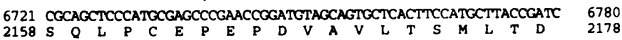

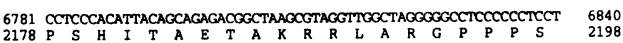

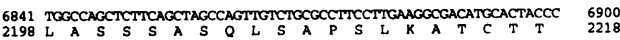

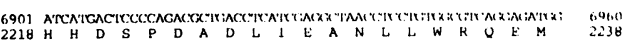

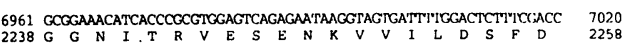

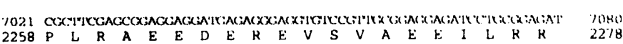

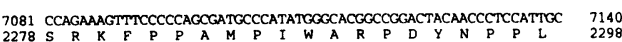

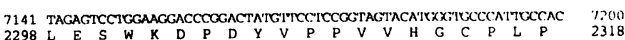

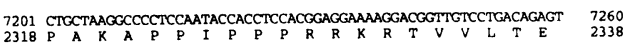

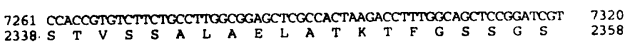

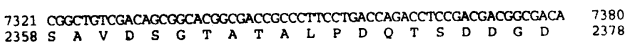

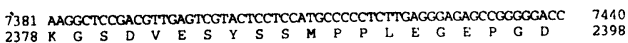

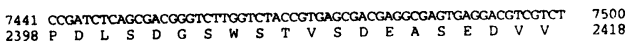

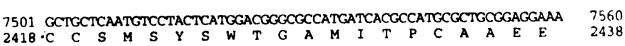

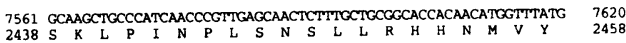

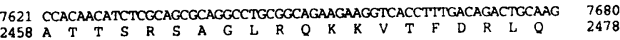

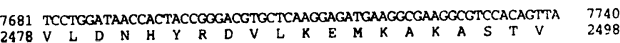

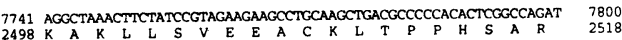

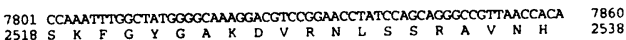

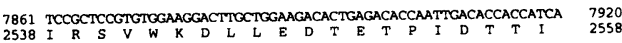

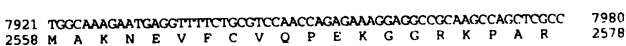

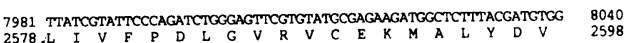

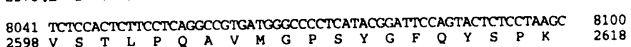

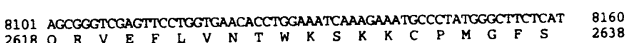

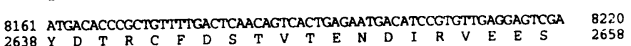

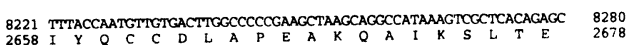

Figure 2

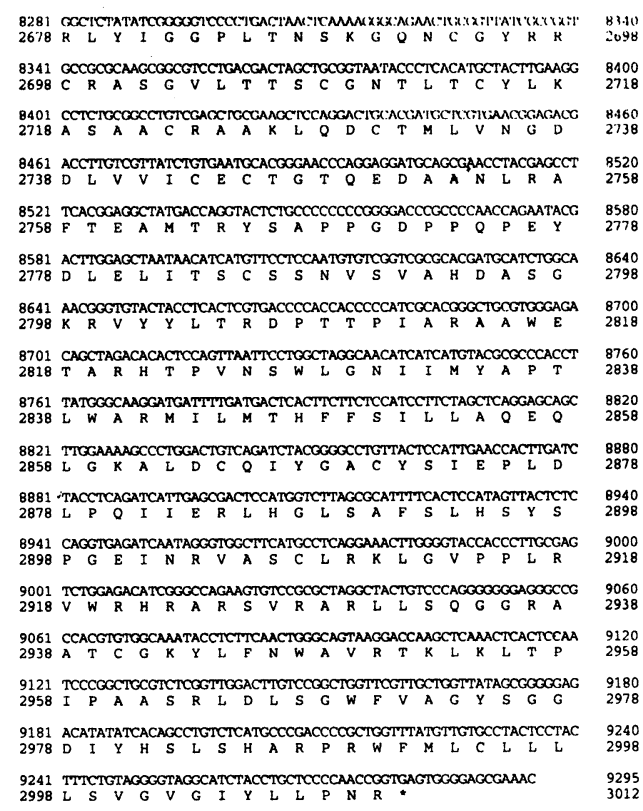

Figure 2

respectively. The sequence homology of $\mathrm{HCV}-\mathrm{N}$ also showed its closer relationship to $\mathrm{HCV}-1$ than to $\mathrm{HC}-\mathrm{J} 6$ and $\mathrm{HC}-\mathrm{J} 8$ isolates (Table 1 ). $\mathrm{HC}-\mathrm{J} 8$ possessed $23 \%$ divergence with $\mathrm{HC}-\mathrm{J} 6$ and has been clearly identified as a separate genotype from HC-J6 (Okamoto et al., 1992b). In order to confirm this conclusion, phylogenetic trees were constructed base on nucleotide

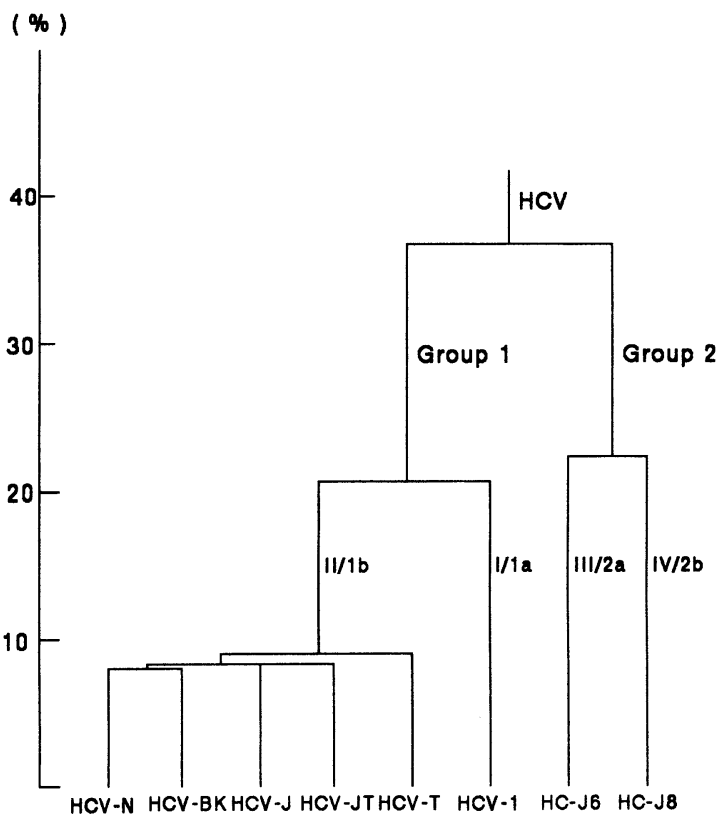

Figure 3 Phylogenetic trees are based on nucleotide divergence (\%). Sequence of all $8 \mathrm{HCV}$ strains were campared and analyzed by the nearest neighbor method. 
divergence throughout entire sequence using nearest neighbor method (Williams and Lance 1977) (Fig. 3). This result indicated that $8 \mathrm{HCV}$ strains can be classified into 2 groups: the group 1 contains genotype II/1b ( HCV-N, HCV-BK, HCV-J, HCV-JT, HCV-T) and genotype $\mathrm{I} / 1 \mathrm{a}$ (HCV-1); while group 2 contains genotype III/2a (HC-J6) and genotype IV/2b ( HC-J8).

Sequence variation in the $\mathrm{E} 1$ and $\mathrm{N}$-terminus of $\mathrm{E2} /$ NS1 envelope glycoprotein regions among $3 \mathrm{HCV}$ Nagasaki strains and 7 other HCV strains with published sequences

Recently, many papers reported that hypervariable regions existed in the $\mathrm{N}$-terminus of E2/NS1 envelope glycoprotein region of $\mathrm{HCV}$ genome (Hijikata et al., 1991; Weiner et al., 1991; Honda et al., 1993). In order

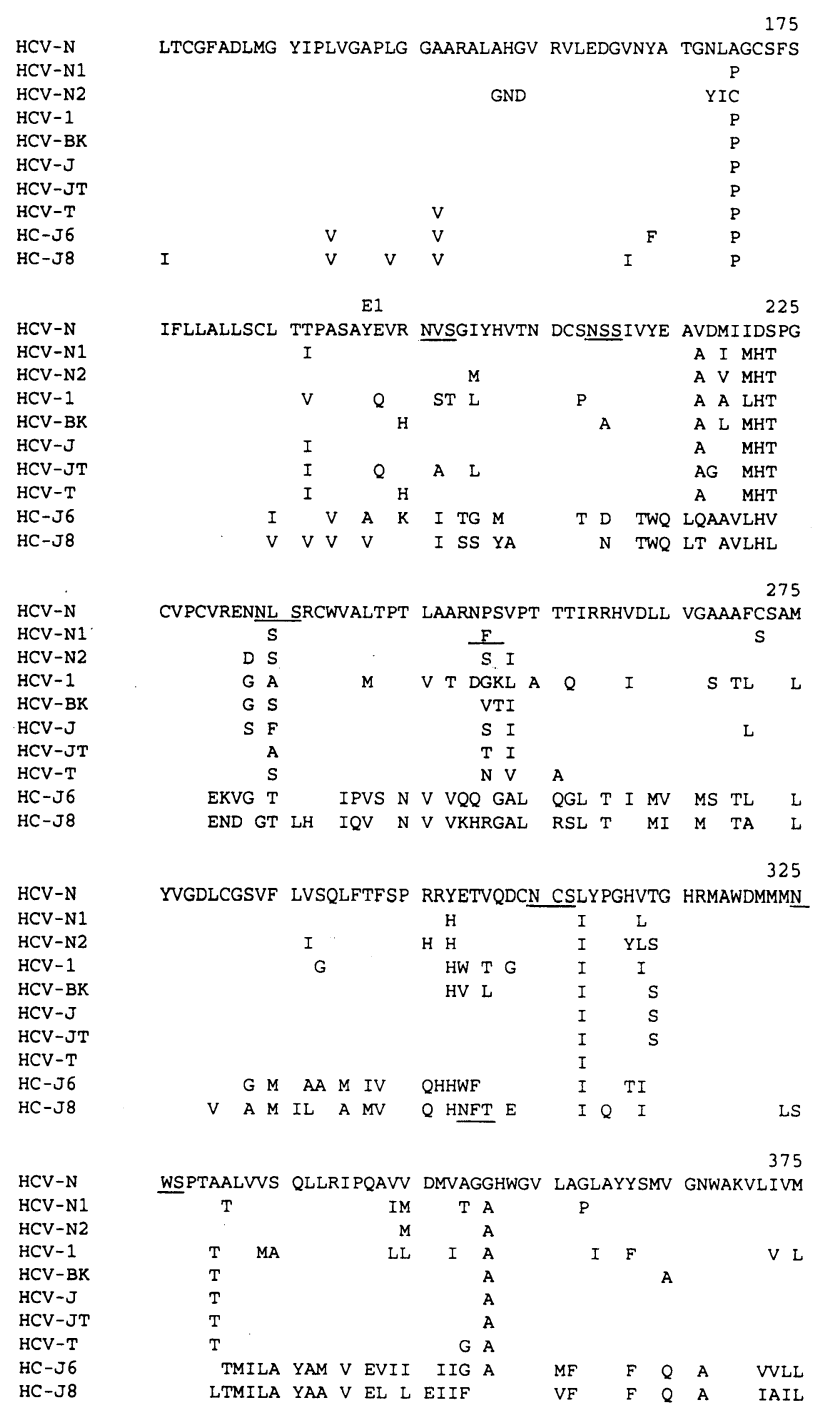

to know the HCV sequence diversity of the envelope glycoprotein in local area of Nagasaki, 2 other $\mathrm{HCV}$ Nagasaki strains (HCV-N1 and HCV-N2) were also cloned and sequenced for the region of nt 623-1988, aa 126-580 (nt and aa base on the HCV-N number). Alignment comparison of deduced aa sequence among 3 Nagasaki strains and other $7 \mathrm{HCV}$ strains with published sequences were shown in Fig. 4. Two hypervariable regions (HVR) were discovered in the $\mathrm{N}$-terminus of E2/NS1 region. HVR 1 (aa 384-411) was located directly downstream at the beginning of the E2/NS1, whereas HVR 2 (aa 475-480) was observed 64 aa downstream from the HVR 1 . In the HVR 1 consisting of 28 aa, 10 aa residues were well-conserved among 3 Nagasaki strains. Whereas only 3 aa were conserved in this

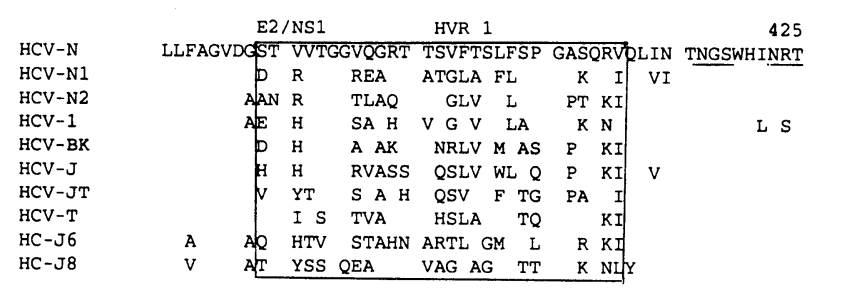

\begin{tabular}{|c|c|c|c|c|c|c|c|c|c|c|c|}
\hline $\mathrm{HCV}-\mathrm{N}$ & ALNCNDS & LHT & GFL & AGVF & YHN $\mathrm{Y}$ & KFNSSC & GCPER & LATC & RPIDKF & DQG & $\begin{array}{r}474 \\
\text { WGPITY }\end{array}$ \\
\hline $\mathrm{HCV}-\mathrm{N} 1$ & $\bar{E}$ & QA & L & $\mathrm{AL}$ & TY N & $\mathrm{N}$ & $\mathrm{T}$ & $M Q$ & $\mathrm{~N}$ & & $\mathrm{~N}$ \\
\hline $\mathrm{HCV}-\mathrm{N} 2$ & & $\dot{Q}$ & & $\mathrm{AL}$ & $\mathrm{VH}$ & & & M S & $s$ & & \\
\hline $\mathrm{HCV}-1$ & & $\mathrm{~N}$ & w & L & $\mathrm{H}$ & & & $\mathrm{s}$ & LTD & & $\mathrm{s}$ \\
\hline $\mathrm{HCV}-\mathrm{BK}$ & & $Q$ & & AL & TH S & & & $M Q$ & $\mathrm{~T}$ & & \\
\hline HCV-J & & $Q$ & I & AL & AH $\mathrm{R}$ & A & & M S & E & A & $\mathrm{H}$ \\
\hline HCV-JT & E & N & F & AL & $\mathrm{AH}$ & & & M S & SS & A & \\
\hline $\mathrm{HCV}-\mathrm{T}$ & & $Q$ & & $\mathrm{SL}$ & $A H R$ & A & & $\mathrm{MS}$ & $\mathrm{s}$ & & \\
\hline $\mathrm{HC}-\mathrm{J} 6$ & & & & SL & $\mathrm{TH} \mathrm{S}$ & $\mathrm{s}$ & & MSA & $S$ EA & RV & \\
\hline $\mathrm{HC}-\mathrm{J} 8$ & & $Q$ & & SL & $\mathrm{TH}$ & & & SS & GL D & RI & TLE E \\
\hline
\end{tabular}

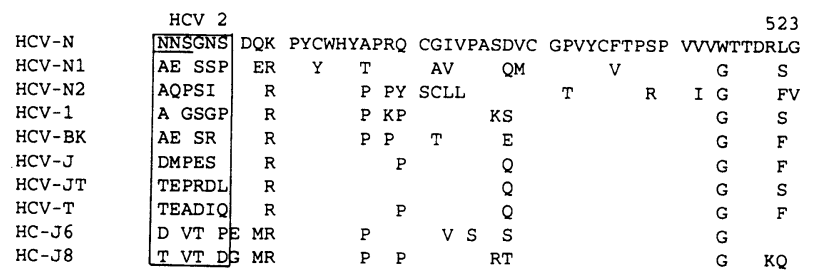

573

HCV-N VPTYTWGENE TDVLLLNNTR PPHGNWFGCT WMNSTGFTKT CGGPPCNIGG $\mathrm{HCV}-\mathrm{N} 1$ $\mathrm{HCV}-\mathrm{N} 2$ $\mathrm{HCV}-1$ $\mathrm{HCV}-\mathrm{BK}$ HCV-J HCV-JT HCV $-\mathrm{T}$ HC-J6

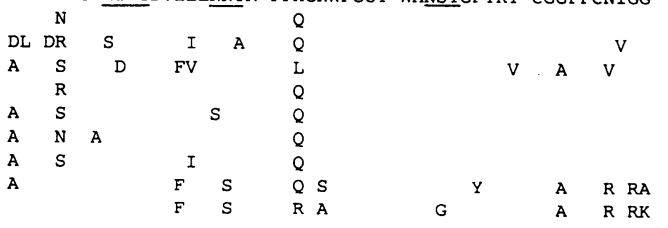

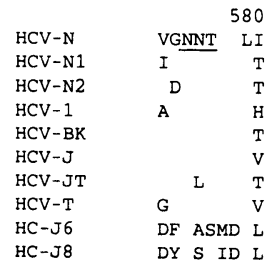

Figure 4 Alignment of amino acid sequence among $10 \mathrm{HCV}$ strains (HCV-N, HCV-N1, HCV-N2, HCV-1, HCV $-\mathrm{BK}, \mathrm{HCV}-\mathrm{J}, \mathrm{HCV}-\mathrm{JT}, \mathrm{HCV}-\mathrm{T}, \mathrm{HC}-\mathrm{J} 6$ and $\mathrm{HC}-\mathrm{J} 8$ ) in the $\mathrm{E} 1$ and $\mathrm{N}$-terminus of E2/NS1 regions (aa 126-580). Two hypervariable regions (HVR 1 and HVR 2) are shown by boxes. Potential $\mathrm{N}$ -glycosylation sites $(\mathrm{N}-\mathrm{X}-\mathrm{S} / \mathrm{T})$ are underlined. 
Table $2 \mathrm{HCV}$ genotype classification including HCV-N from this study and 15 entire HCV strains which have been reported by Sakamoto et al. (1994).

\begin{tabular}{|c|c|c|c|c|c|c|}
\hline Group & \multicolumn{3}{|c|}{ Group 1} & \multicolumn{2}{|c|}{ Group 2} & Group 3 \\
\hline Genotype & $\mathrm{I} / 1 \mathrm{a}$ & I c & $\mathrm{II} / 1 \mathrm{~b}$ & $\mathrm{III} / 2 \mathrm{a}$ & $\mathrm{IV} / 2 \mathrm{~b}$ & $\mathrm{~V} / 3 \mathrm{a}$ \\
\hline $\mathrm{HCV}$ strains & $\begin{array}{l}\mathrm{HCV}-1 \\
\mathrm{HCV}-\mathrm{H} \\
\mathrm{HC}-\mathrm{J} 1\end{array}$ & HC-G9 & $\begin{array}{l}\text { HCV-J } \\
\text { HCV-BK } \\
\text { HC-J4/83 } \\
\text { HC-J4/91 } \\
\text { HCV-JT } \\
\text { HCV-T } \\
\text { HCV-JK1 } \\
\text { HC-C2 } \\
\text { HCV-N }\end{array}$ & $\mathrm{HC}-\mathrm{J} 6$ & $\mathrm{HC}-\mathrm{J} 8$ & NZL1 \\
\hline $\begin{array}{l}\text { Geographic } \\
\text { Area }\end{array}$ & $\begin{array}{l}\text { U.S.A. } \\
\text { Japan }\end{array}$ & Indonesian & $\begin{array}{l}\text { Japan } \\
\text { China } \\
\text { Taiwan }\end{array}$ & Japan & Japan & $\begin{array}{l}\text { New } \\
\text { Zealand }\end{array}$ \\
\hline
\end{tabular}

region when a total $10 \mathrm{HCV}$ strains were compared. In the HVR 2 consisting of 6 aa, none of the aa were conserved among 3 Nagasaki strains. This hypervariability was also observed when a total $10 \mathrm{HCV}$ strains were compared simultaneously. In the $\mathrm{E} 1$ and $\mathrm{N}$-terminus of the E2/NS1 regions, 2 well conserved aa sequences were observed among $10 \mathrm{HCV}$ isolates: $\mathrm{G}-\mathrm{H}$ -R-M-A-W-D-M-M (aa 315-323) and W-F-G-C-T-W $-\mathrm{M}-\mathrm{N}$ (aa 549-556). Although $14 \mathrm{~N}$-glycosylation sites $(\mathrm{N}-\mathrm{X}-\mathrm{S} / \mathrm{T})$ were identified in $\mathrm{HCV}-\mathrm{N}$, one of them (NNS at aa No. 475-477) was unique to this strain and not present in 2 other Nagasaki strains and 7 other $\mathrm{HCV}$ strains with published sequences. Instead, $\mathrm{HCV}-\mathrm{N} 1$ and HCV-N2 possessed another N-glycosylation site (NFS or NSS at aa No. 250-252). This glycosylation site is found also in HCV-BK, HCV-J, HCV-JT, and HCV-T (Fig. 4). Altogether, only $9 \mathrm{~N}$-glycosylation sites were conserved in the $\mathrm{E} 1$ and $\mathrm{N}$-terminal of the E2/NS1 regions among $10 \mathrm{HCV}$ strains shown in Fig. 4.

\section{DISCUSSION}

Relationship between genotype and geographic area of $\mathrm{HCV}$ strains including $\mathrm{HCV}-\mathrm{N}$ was shown by their sequence comparison using the classification by Sakamoto et al. (1994) (Table 2). The sequence homology indicated that $\mathrm{HCV}-\mathrm{N}$ strain did not possess its local genotypic character, and similar to $\mathrm{HCV}-\mathrm{BK}, \mathrm{HCV}-\mathrm{J}$ and HCV-JT strain which were isolated from other areas of Japan. All these strains apparently belong to the same genotype II/1b of group 1 . This genotype also included $\mathrm{HC}-\mathrm{J} 4 / 83$; HC-J4/91; HCV-JK1 of Japanese $\mathrm{HCV}$ strains, $\mathrm{HC}-\mathrm{C} 2$ of Chinese $\mathrm{HCV}$ strain and $\mathrm{HCV}$ - $\mathrm{T}$ of Taiwan strain. The genotype $1 \mathrm{c}$ of group 1 includes $\mathrm{HC}-\mathrm{G} 9$ which was derived from Indonesian strain. While, the genotype $\mathrm{I} / 1$ a of group 1 includes $\mathrm{HCV}-1$ and $\mathrm{HCV}-\mathrm{H}$ which were derived from American strains. Although another $\mathrm{HC}-\mathrm{J} 1$ was Japanese strain, it was supposed to have originated from the US, because $\mathrm{HC}-\mathrm{J} 1$ was derived from a Japanese haemophiliac who developed hepatitis $\mathrm{C}$ after receiving US-made factor VIII (Okamoto et al., 1992a). In group 2, HC-J6 and HC -J8 belong to the III/2a and the IV/2b genotype, respectively. Both of them were Japanese strains. In group 3 , NZL1 that came from New Zealand belongs to V/3a genotype (Table 2). Simmonds et al. (1993b) have classified 6 major genotypes of $\mathrm{HCV}$ from $76 \mathrm{HCV}$ isolates, which were almost worldwide collection, using phylogenetic analysis of the NS5 region. The $76 \mathrm{HCV}$ isolates contained entire sequence of $\mathrm{HCV}-1, \mathrm{HCV}-\mathrm{H}$, $\mathrm{HCV}-\mathrm{J}, \mathrm{HCV}-\mathrm{BK}, \mathrm{HCV}-\mathrm{T}, \mathrm{HCV}-\mathrm{JT}, \mathrm{HC}-\mathrm{J} 6$ and $\mathrm{HC}$ - J8 strains and other partial sequence of $\mathrm{HCV}$ strains. From this classification, only genotype 4, 5 and 6 showed highly restricted geographical distributions, being apparently confined to Egypt, South Africa and Hong Kong respectively (Simmonds et al., 1993b).

Two hypervariable regions have already been observed in the $\mathrm{N}$-terminus of the $\mathrm{E} 2 / \mathrm{NS} 1$ region. The number of conserved aa in the HVR 1 among 3 Nagasaki strains were higher than among a total 10 strains including 3 Nagasaki strains and 7 other HCV strains with published sequences. The numbers of conserved aa (5 aa/28 aa) among HCV strains of genogroup 1 (HCV -N/HCV-1/HCV-BK/HCV-J/HCV-JT/HCV-T) were higher than those (3 aa/28 aa) among $10 \mathrm{HCV}$ strains which included genogroup 2 (HC-J6 and $\mathrm{HC}-\mathrm{J} 8$ ). This result may give us an idea that aa conservation in the $\mathrm{HVR} 1$ of the same genotype or genogroup of $\mathrm{HCV}$ are 
higher than among the different genotype or genogroup of HCV strains. Therefore, the 3 Nagasaki HCV strains may have been originated from the same ancestor.

In contrast, none of the aa was conserved among 3 Nagasaki strains nor among a total $10 \mathrm{HCV}$ strains in the HVR 2 which showed higher variability than the HVR 1. The reason why mutations occur so frequently in only limited regions such as HVR is not known (Tanaka et al., 1992). The higher degree of divergence in $\mathrm{HCV} \mathrm{E2/NS1} \mathrm{region} \mathrm{might} \mathrm{reflect} \mathrm{the} \mathrm{immune} \mathrm{selec-}$ tion and suggest that this region cannot probably be an ideal target for future vaccine development.

Regarding the $\mathrm{N}$-glycosylation sites in the $\mathrm{E} 1$ and $\mathrm{N}$ -terminus of the E2/NS1 region, only 9 sites were conserved out of the 14 sites seen in $\mathrm{HCV}-\mathrm{N}$. Some of the glycosylation sites, therefore, would not be essential for the survival, transmission and maintenance of $\mathrm{HCV}$ in nature. On the other hand, 2 well-conserved aa sequences were found in the $\mathrm{E} 1$ and $\mathrm{N}$-terminus of the E2/NS1 regions among the $10 \mathrm{HCV}$ strains compared. These conserved sequences may be better targets of vaccine development if they were related with protective immunity.

The nt sequence homology of HCV-N in the 5'UTR shows high conservation comparing with other $7 \mathrm{HCV}$ isolates (nt $>91.5 \%$ ) and was used as an ideal target for PCR amplification to detect HCV RNA (Okamoto et al., 1990). But recently, some papers reported that several HCV strains have sequence variation in the 5' UTR (Lee et al., 1992; Bukh et al., 1992; Simmonds et al., 1993a). Therefore additional informations would be required to select optimal primer for PCR diagnosis on $\mathrm{HCV}$. The functional motifs of the putative encoded proteins of HCV (Plagemann et al., 1991; Tanaka et al., 1992) have also been found in $\mathrm{HCV}-\mathrm{N}$. There are consensus sequences of RNA helicase in the NS3 region: Gly-Ser-Gly-Lys-Ser-Thr (aa 1233-1238) and Gln-Arg -Arg-Gly-Arg-Thr-Gly-Arg (aa 1486-1493), while NS5 region possessed consensus sequence of RNA-dependent RNA polymerase: Gly-Asp-Asp (aa 2736-2738). The sequence heterogeneity in putative structural proteins and nostructural proteins of HCV provided significant evidence for genotype classification. The genetic informations of $\mathrm{HCV}-\mathrm{N}$ genome obtained in this study will be useful in future understanding on the pathogenesis, diagnosis and development of vaccine for $\mathrm{HCV}$.

\section{ACKNOWLEDGEMENTS}

The author would like to express thanks to Prof. A. Igarashi for valuable advice and encouragement, careful reading and comments of the manuscript.

Special thanks are due to Dr. K. Morita, Dr. S. Kurihara, Dr. K.Z. Thant and Dr. Y. Tsuda for useful suggestion and kindly assistance.

This study was supported by Health Science Research Grants (Non A-NonB Hepatitis Research Grants) from the Ministry of Health and Welfare of Japan.

\section{REFERENCES}

1) Bukh, J., Purcell, R.H. and Miller, R.H. (1992): Sequence analysis of the 5 ' noncoding region of hepatits C virus. Proc. Natl. Acad. Sci. U.S.A., 89, 4942-4946

2 ) Chen, P.J., Lin, M.H., Tai, K.F., Liu, P.C., Lin, C.J. and Chen, D.S. (1992): The Taiwanese hepatitis C virus genome: sequence determination and mapping the 5 termini of genomic and antigenomic RNA. Virology, $188,102-113$

3 ) Choo, Q.L., Kuo, G., Weiner, A.J., Overby, L.R., Bradley, D.W. and Houghton, M. (1989): Isolation of a cDNA clone derived from a blood-borne non- $\mathrm{A}$, non-B viral hepatitis genome. Science, 244, 359-362

4 ) Choo, Q.L., Richman, K.H., Han, J.H., Berger, K., Lee, C., Dong, C., Gallegos, C., Coit, D., Medina-Selby, A., Barr, P.J., Weiner, A.J., Bradley, D.W., Kuo, G. and Houghton, M. (1991): Genetic organization and diversity of the hepatitis C virus. Proc. Natl. Acad. Sci. U.S. A., 88, 2451-2455

5 ) Hijikata, M., Kato, N., Ootsuyama, Y., Nakagawa, M., Ohkoshi, S. and Shimotohno, K. (1991): Hypervarible regions in the Putative Glycoprotein of Hepatitis C virus. Biochem. Biophys. Res. Commun., 175, 220-228

6 ) Honda, M., Kaneko, S., Unoura, M., Kobayashi, K. and Murakami, S. (1992): Sequence comparisons for a hepatitis $\mathrm{C}$ virus genome RNA isolated from a patient with liver cirrhosis. Gene, 120, 317-318

7 ) Honda, M., Kaneko, S., Unoura, M., Kobayashi, K. and Murakami, S. (1993): Sequence analysis of putative structural regions of hepatitis $\mathrm{C}$ virus isolated from 5 Japanese patient with hepatocellular carcinoma. Arch Virol., 128, 163-169

8 ) Inchauspe, G., Zebedee, S., Lee, D.H., Sugitani, M. and Nasoff, M. (1991): Genomic structure of the human prototype strain $\mathrm{H}$ of hepatitis $\mathrm{C}$ virus: comparison with American and Japanese isolates. Proc. Natl. Acad. Sci. U.S.A., 88, 10292-10296

9 ) Kato, N., Hijikata, M., Ootsuyama, Y., Nakagawa, M., Ohkoshi, S., Sugimura, T. and Shimotohno, K. (1990): Molecular cloning of the human hepatitis $\mathrm{C}$ virus genome from Japanese patient with non-A, non-B hepatitis. Proc. Natl. Acad. Sci. U.S.A., 87, 9524-9528

10) Kurihara, S. (1992): Comparative nucleotide and amino acid sequences of the envelope protein of five hepatitis $\mathrm{C}$ viral cDNA clones isolated from $\mathrm{HCV}$ infected patients 
in Nagasaki Prefecture. Trop. Med., 34, 39-54

11) Lee, C.H., Cheng, C., Wang, J. and Lumeng, L. (1992): Identification of hepatitis $\mathrm{C}$ viruses with a nonconserved sequence of the 5 ' untranslated region. J. Clin. Microbiol., 1602-1604

12) Okamoto, H., Okada, S., Sugiyama, Y., Tanaka, T., Sugai, Y., Akahane, Y., Machida, A., Mishiro, S., Yoshizawa, H., Miyakawa, Y. and Mayumi, M. (1990): Detection of hepatitis $\mathrm{C}$ virus RNA by a two-stage polymerase china reaction with two pairs of primers deduced from the 5'-noncoding region. Japan. J. Exp. Med., 60, 215-222

13) Okamoto, H., Okada, S., Sugiyama, Y., Kurai, K., Iizuka, H., Machida, A., Miyakawa, Y. and Mayumi, M. (1991): Nucleotide sequence of the genomic RNA of hepatitis C virus isolated from a human carrier: comparison with reported isolates for conserved and divergent regions. J. Gen. Virol., 72, 2697-2704

14) Okamoto, H., Kanai, N. and Mishiro, S. (1992a): Full -length nucleotide sequence of a Japanese hepatitis C virus isolate (HC-J1) with high homology to USA isolates. Nucleic Acids Res., 20, 6410

15) Okamoto, H., Kurai, K., Okada, S., Yamamoto, K., Iizuka, H., Tanaka, T., Fukuda, S., Tsuda, F. and Mishiro, S. (1992b): Full-length sequence of a hepatitis $\mathrm{C}$ virus genome having poor homology to reported isolates: comparative study of four distinct genotypes. Virology, 188, 331-341

16) Okamoto, H., Kojima, M., Okada, S., Yoshizawa, H., Iizuka, H., Tanaka, T., Muchmore, E.E., Peterson, D.A., Ito, Y. and Mishiro, S. (1992c): Genetic drift of hepatitis $\mathrm{C}$ virus during an 8.2-year infection in a chimpanzee: variability and stability. Virology 190, 894-899

17) Okamoto, H., Kojima, M., Sakamoto, M., Iizuka, H., Hadiwandowo, S., Suwignyo, S., Miyakawa, Y. and Mayumi, M. (1994): The entire nucleotide sequence and classification of a hepatitis $\mathrm{C}$ virus isolate of a novel genotyoe from an Indonesian patient with chronic liver disease. J. Gen. Virol., 75, 629-635

18) Plagemann, P.G.W. (1991): Hepatitis C virus (Brief Review). Arch Virol., 120, 165-180
19) Sakamoto, M., Akahane, Y., Tsuda, F., Tanaka, T., Woodfield, D.G. and Okamoto, H. (1994): Entire nucleotide sequence and characterization of a hepatitis $\mathrm{C}$ virus of genotype V/3a. J. Gen. Virol., 75, 1761-1768

20) Simmonds, P., McOmish, F., Yap, P.L., Chan, S.-W., Lin, C.K., Dusheiko, G., Saeed, A.A. and Holmes, E.C. (1993a): Sequence variability in the $5^{\prime}$ non-coding region of hepatitis $\mathrm{C}$ virus: identification of a new virus type and restrictions on sequence diversity. J. Gen. Virol., 74, 661-668

21) Simmonds, P., Holmes, E.C., Cha, T.-A., Chan, S-W., McOmish, F., Irvine, B., Beall, E., Yap, P.L., Kolberg, J. and Urdea, M.S. (1993b): Classification of hepatitis C virus into six major genotypes and a series of subtypes by phylogenetic analysis of the NS-5 region. J. Gen. Virol., 74, 2391-2399

22) Tanata, T., Kato, N., Nakagawa, M., Ootsuyama, Y., Cho, M-J., Nakazawa, T., Hijikata, M., Ishimura, Y. and Shimotohno, K. (1992): Molecular cloning of hepatitis C virus genome from a single Japanese carrier: sequence variation within the same individual and among infected individuals. Virus Res., 23, 39-53

23) Takamizawa, A., Mori, C., Fuke, I., Manabe, S., Murakami, S., Fujita, J., Onishi, E., Andoh, T., Yoshida, I. and Okayama, H. (1991): Structure and organization of the hepatitis $\mathrm{C}$ virus genome isolated from human carriers. J. Virol., 65, 1105-1113

24) Wang, Y., Okamoto, H., Tsuda, F., Nagayama, R., Tao, Q.M. and Mishiro, S. (1993): Prevalence, genotypes, and an isolate $\mathrm{HC}-\mathrm{C} 2$ of hepatitis $\mathrm{C}$ virus in Chinese patients with liver disease. J. Med. Virol., 40, 254-260

25) Weiner, A.J., Brauer, M.J., Rosenblatt, J., Richman, K. H., Tung, J., Crawford, K., Bonino, F., Saracco, G., Choo, Q.L., Houghton, M. and Han, J.H. (1991): Variable and hypervariable domains are found in the regions of $\mathrm{HCV}$ corresponding to the flavivirus envelope and NS1 proteins and pestivirus envelope glycoproteins. Virology, $180,842-848$

26) Williams, W.T. and Lance, G.N. (1977): Statistical methods for digital computers: hierarchical classificatory methods. 269-295 